University of Nebraska - Lincoln

DigitalCommons@University of Nebraska - Lincoln

January 1970

\title{
Projected Hartree Product Wavefunctions. IV. Radial Correlation in Some Three- and Four-Electron Atoms
}

L.G. Heikes

University of Nebraska - Lincoln

Gordon A. Gallup

UNL,ggallup1@unl.edu

Follow this and additional works at: https://digitalcommons.unl.edu/physicsgallup

Part of the Physics Commons

Heikes, L.G. and Gallup, Gordon A., "Projected Hartree Product Wavefunctions. IV. Radial Correlation in Some Three- and Four-Electron Atoms" (1970). Gordon Gallup Publications. 20.

https://digitalcommons.unl.edu/physicsgallup/20

This Article is brought to you for free and open access by the Research Papers in Physics and Astronomy at DigitalCommons@University of Nebraska - Lincoln. It has been accepted for inclusion in Gordon Gallup Publications by an authorized administrator of DigitalCommons@University of Nebraska - Lincoln. 


\title{
Projected Hartree Product Wavefunctions. IV. Radial Correlation in Some Three- and Four-Electron Atoms*
}

\author{
L. G. Heikest and G. A. Galiur \\ Department of Chemistry, University of Nebraska, Lincoln, Nebraska 68506
}

(Received 7 April 1969)

\begin{abstract}
The energies of some three- and four-electron atoms including radial correlation have been determined by a spin-free version of the projected Hartree-Fock method. The wavefunction possesses the maximum flexibility allowable under the general restrictions of the method, and it is shown that this flexibility is necessary to adequately account for the differences between intra-and intershell correlation. Some comments on the AMO method are included.
\end{abstract}

\section{INTRODUCTION}

In articles $\mathrm{I}^{1}$ and $\mathrm{II}^{2}$ of this series a method is described for using the theory of symmetric groups in doing a projected Hartree-Fock ${ }^{3}$ (optimization after projection) treatment of an atomic or molecular system. In general, the projection involves both spatial and identity symmetries, and such a treatment was given for $\mathrm{H}_{2}$ in III. ${ }^{4}$ However, we may study the effect on the correlation energy of various factors in the treatment without the added complication of spatial symmetry projections, by looking at radial correlation in some simple atoms.

The present method, a different-orbitals-for-differentspins (DODS) calculation, is an extension of the independent particle model, in which the wavefunction is given as a projected Hartree product (PHP) made up of $n$ single-particle spatial orbitals for $n$ electrons, that is

$$
\phi=\prod_{i=1}^{n} u_{i}(i) \text {. }
$$

The energy for a pure spin state of $S$ may be obtained from a primitive idempotent of the group algebra of $S_{n}$ associated with the Young tableau $\left[2^{(n / 2)-s}, 1^{2 S}\right]=$ $[\mu], e^{(\mu)}$. Thus, we have,

$$
E=\left\langle\phi\left|H e^{(\mu)}\right| \phi\right\rangle /\left\langle\phi\left|e^{(\mu)}\right| \phi\right\rangle,
$$

for a spin-free Hamiltonian, $H$. As was pointed out in II, where the $f_{\mu}$ standard tableaux of shape $[\mu]$ and if $\pi_{1}(=I), \pi_{2}, \cdots, \pi_{f}$, are the permutations giving the $i$ th tableau from the first, we may usually improve the energy expression by replacing Eq. (2) by

where

$$
E=\left\langle\psi\left|H e^{(\mu)}\right| \psi\right\rangle\left\langle\left\langle\psi\left|e^{(\mu)}\right| \psi\right\rangle,\right.
$$

$$
\psi=\sum_{i=1}^{f} \gamma_{i} \pi_{i} \phi
$$

\footnotetext{
* Based in part on the thesis presented by L. G. Heikes in partial fulfillment of the Ph.D. degree.

+ NSF Predoctoral Fellowship 1966-1968, NSF Traineeship 1968-1969.

1 G. A. Gallup, J. Chem. Phys. 48, 1752 (1968).

${ }^{2}$ G. A. Gallup, J. Chem. Phys. 50, 1206 (1969).

3 P.-O. Löwdin Phys. Rev, 97, 1509 (1955).

4 R. C. Morrison and G. A. Gallup, J. Chem. Phys. 50, 1214 (1969).
}

In our procedure the energy $E$ is minimized with respect to the $\gamma_{i}$ in addition to the orbitals, $u_{i}$. As we shall see, the values of the $\gamma_{i}$ 's can have a profound effect on the way the PHP method produces correlation.

Matsen et al. ${ }^{5}$ have used a formulation of the energy expression very similar to Eq. (4). Goddard ${ }^{6}$ and Kaldor, Schaefer, and Harris ${ }^{7}$ have given equivalent treatments of some simple atoms which is equivalent to the present one if a certain fixed set of $\gamma_{i}^{\prime}$ 's is used. (a)

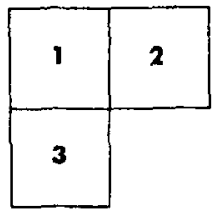

(b)

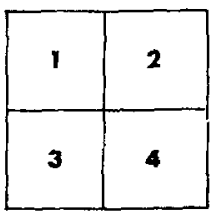

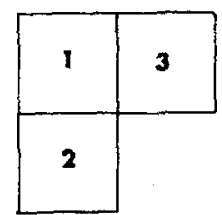

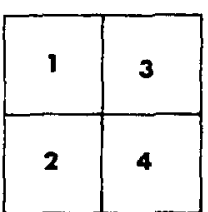

FIG. 1. Standard tableaux for (a) three-electron systems and (b) four-electron systems.

Ritter, Pauncz, and Appel ${ }^{8}$ have given a treatment of $\mathrm{Li}$ equivalent to the present calculation with a fixed set of $\gamma_{i}$ 's different from Goddard's choice. More recently, Lunell ${ }^{9}$ has given a calculation of $\mathrm{Li}$ in which the $\gamma_{i}$ 's are optimized, but the orbitals are but partially optimized.

${ }^{5}$ F. A. Matsen, Advan. Quantum Chem. 1, 59 (1964); J. Phys. Chem. 68, 3282 (1964); 70, 1568 (1966); F. A. Matsen, A. A. Cantu, and R. D. Poshusta, ibid. 70, 1558 (1966).

${ }^{6}$ W. A. Goddard, Phys. Rev. 157, 73, 81, 93 (1967); J. Chem. Phys. 48, 1008 (1968).

${ }^{7}$ U. Kaldor, H. F. Schaefer, and F. E. Harris, Intern. J. Quantum Chem. 2S, 13 (1968).

${ }^{8}$ Z. W. Ritter, R. Pauncz, and K. Appel, J. Chem. Phys. 35, 571 (1961).

${ }^{9}$ S. Lunell, Phys. Rev. 173, 85 (1968). 
These previous calculations on the simple atoms include the possibility of radial correlation only, and as was mentioned above, we shall make a similar restriction in the present treatment.

\section{THREE-ELECTRON ATOMS}

As was pointed out in II, we seem to have fewer computational difficulties by using $\theta N P N$ for $e^{(\mu)}$ than by using $\theta^{\prime} P N P$ and this choice is taken. There are three orbitals in these atoms, which shall be designated $a, b$, and $c$. Thus

$$
\phi=a(1) b(2) c(3),
$$

and each of these orbitals is represented as a linear combination of STO's. Table I gives the principle quantum number and the fully optimized $\zeta$ 's for the three-electron atoms considered here. Figure 1(a) shows the two standard tableaux for doublet states of three electrons, from which it is seen that $\pi_{1}=I, \pi_{2}=(23)$, and hence

$$
e^{(2,1)} \psi=e^{(2,1)}\left[\gamma_{1} a(1) b(2) c(3)+\gamma_{2} a(1) c(2) b(3)\right] .
$$

The choices among the several alternatives we have made in setting up Eq. (6), now forces $a$ to be the $2 s$-like orbital and $b$ and $c$ to be the two $1 s$-like orbitals. Table II gives the energies, orbital coefficients, and the $\gamma_{i}$ 's for each of the nuclear charges considered. Roothaan, Sachs, and Weiss ${ }^{10}$ have given restricted SCF energies for these systems, and the correlation energies are also shown. These results are most easily compared with Goddard's, ${ }^{6}$ and it is seen that his restriction $\left(\gamma_{2}=0\right)$ prevents obtaining any considerable correlation energy. The calculation by Pauncz et al. ${ }^{8}$ corresponds to the restriction $\gamma_{1}=\gamma_{2}$, and as can be seen this is very close to the optimal situation.

We have used the iterative solution outlined in I with one exception: The starting orbitals for $b$ and $c$ are obtained from a calculation of the corresponding twoelectron atom first. Then, a third electron is added, and all three orbitals are optimized. If arbitrary starting orbitals are used for a concerted three-electron optimization, it is possible to reach a $c u l$ de sac (but apparently not a local minimum) in the iteration from which this type of first-order iterative procedure cannot

TABLE I. $\zeta$ for three-electron atoms.

\begin{tabular}{lllll}
\hline \hline & $\mathrm{Li}$ & $\mathrm{Be}^{+}$ & $\mathrm{B}^{++}$ & $\mathrm{C}^{+++}$ \\
\hline $1 s$ & 4.56 & 6.01 & 7.61 & 9.10 \\
$2 s$ & 4.13 & 5.50 & 7.01 & 8.10 \\
$1 s$ & 2.69 & 3.52 & 4.46 & 5.40 \\
$2 s$ & 2.12 & 2.81 & 3.61 & 4.30 \\
$1 s$ & 0.795 & 1.01 & 1.28 & 1.60 \\
$2 s$ & 0.617 & 0.811 & $\mathbf{1 . 1 1}$ & 1.30 \\
$2 s$ & 0.279 & 0.211 & 0.251 & 0.310 \\
$3 s$ & 0.099 & 0.111 & 0.136 & 0.160 \\
\hline \hline
\end{tabular}

${ }^{10}$ C. C. J. Roothaan, L. M. Sachs, and A. W. Weiss, Rev. Mod. Phys. 32, 186 (1960).

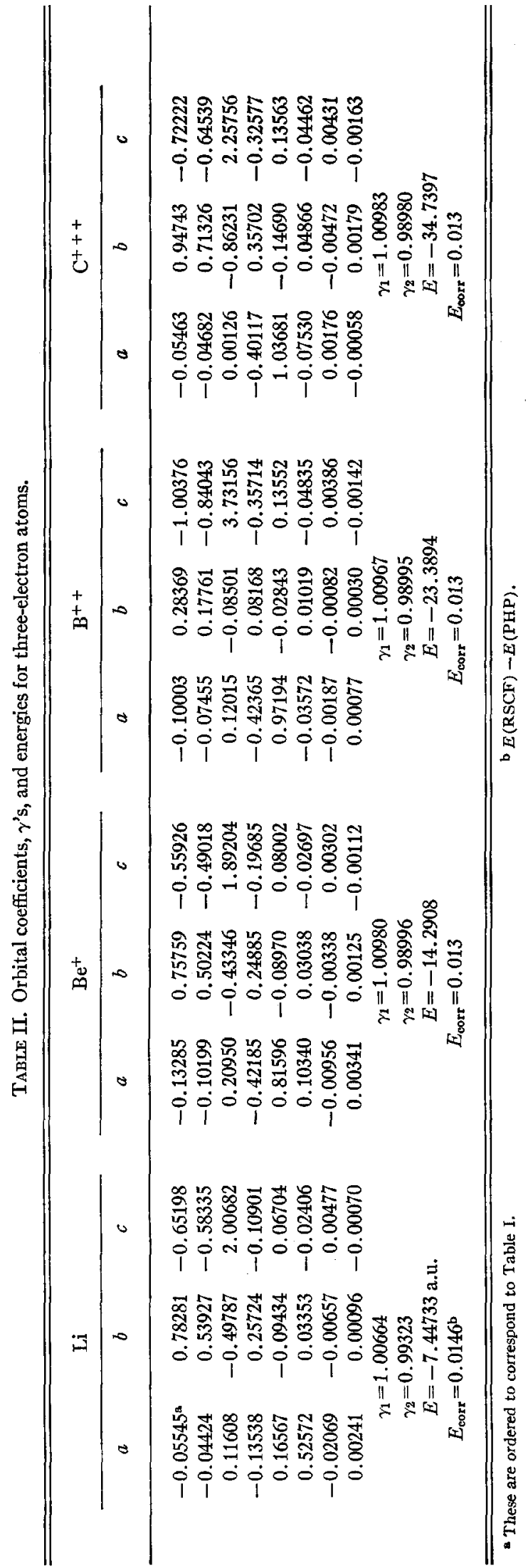


TABLE III. $\zeta$ for four-electron atoms.

\begin{tabular}{llll}
\hline & $\mathrm{Be}$ & $\mathrm{B}^{+}$ & $\mathrm{C}^{++}$ \\
\hline $1 s$ & 6.00 & 7.60 & 9.00 \\
$2 s$ & 5.60 & 7.00 & 8.00 \\
$1 s$ & 3.53 & 4.44 & 5.30 \\
$2 s$ & 2.81 & 3.60 & 4.20 \\
$1 s$ & 1.00 & 1.22 & 1.50 \\
$2 s$ & 0.810 & 1.20 & 1.20 \\
$2 s$ & 0.210 & 0.350 & 0.400 \\
$3 s$ & 0.110 & 0.110 & 0.150 \\
\hline
\end{tabular}

depart in a practical length of time. The above type of "building up" procedure prevents this unhappy occurance, and is very similar to that used by Pauncz et al. ${ }^{8}$ and Lunell. ${ }^{9}$ For a given set of $\zeta$ 's the above procedure for three-electron atoms requires approximately $90 \mathrm{sec}$ on an IBM 360/65 computer. It may be pointed out that a full CI calculation from this basis contains 168 functions.

\section{FOUR-ELECTRON ATOMS}

Figure 1(b) gives the two standard tableaux for four electrons in a singlet state. We designate the four orbitals $a, b, c, d$, and thus

$$
\begin{gathered}
\phi=a(1) b(2) c(3) d(4), \\
\psi=\gamma_{1} a(1) b(2) c(3) d(4)+\gamma_{2} a(1) c(2) b(3) d(4) .
\end{gathered}
$$

Tables III and IV give the results for this case. It is observed that $b$ and $c$ are the $1 s$-like orbitals and $a$ and $d$ the $2 s$-like orbitals. The time here is of the order of 270 sec for one $\zeta$ set, and the corresponding full CI contains 112 functions.

\section{DISCUSSION}

If the results given here are compared with various previous calculations, it is seen that there are a number of interesting points to be investigated. The situations are somewhat different for the cases of three and four electrons, and we take up the smaller case first.

It should be pointed ont that the primitive idempotent $e^{(2,1)}$ contains a factor $[I-(12)]$, and in any term of our wavefunction, the orbitals occupied by electrons 1 and 2 may be orthogonalized to give a term with at most a different numerical factor.

In order to make the analysis it is convenient to introduce two new orbitals,

where

$$
\begin{aligned}
& x=\frac{1}{2}(a+b), \\
& y=\frac{1}{2}(a-b),
\end{aligned}
$$

$$
\langle x \mid y\rangle=0,
$$

if $a$ and $b$ are normalized. Using Eq. (6) it is seen that

$$
\begin{array}{r}
\Phi \equiv e^{(2,1)} \psi=e^{(2,1)}\left\{\left(\gamma_{1}+\gamma_{2}\right)[a(1) x(2) x(3)\right. \\
-a(1) y(2) y(3)]+\left(\gamma_{1}-\gamma_{2}\right)[a(1) x(2) y(3) \\
-a(1) y(2) x(3)]\} .
\end{array}
$$

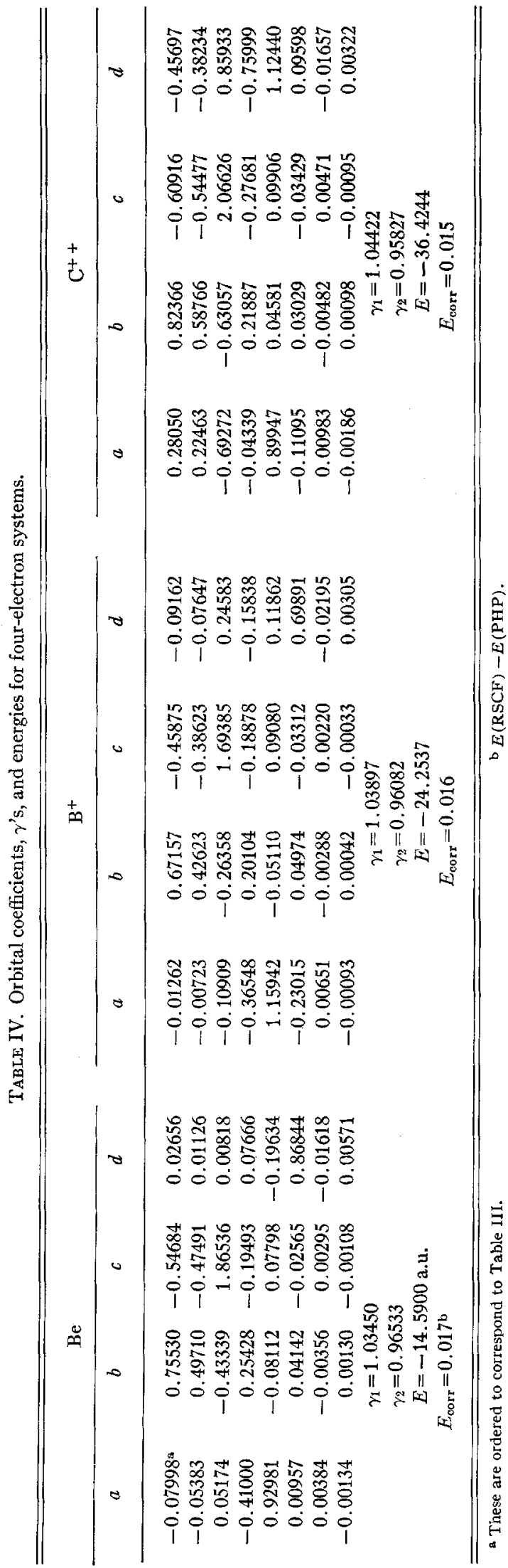


TABLE V. Radial correlation energy for three- and four-electron atoms.

\begin{tabular}{|c|c|c|c|c|c|c|c|}
\hline \multirow{2}{*}{$\begin{array}{l}\text { Type of } \\
\text { calculation }\end{array}$} & \multicolumn{7}{|c|}{ Energy (a.u.) } \\
\hline & $\mathrm{Li}$ & $\mathrm{Be}^{+}$ & $\mathrm{B}^{++}$ & $\mathrm{C}^{+++}$ & $\mathrm{Be}$ & $\mathrm{B}^{+}$ & $\mathrm{C}^{++}$ \\
\hline $\begin{array}{l}\mathrm{PHP}^{\mathbf{a}} \\
\mathrm{CI}^{\mathrm{b}} \\
\text { Exact }^{\mathrm{o}}\end{array}$ & $\begin{array}{l}-0.0146 \\
-0.0444 \\
-0.0453\end{array}$ & $\begin{array}{l}-0.0138 \\
-0.0465 \\
-0.0475\end{array}$ & $\begin{array}{l}-0.0134 \\
-0.0471 \\
-0.0489\end{array}$ & $\begin{array}{l}-0.0136 \\
-0.0477 \\
-0.0498\end{array}$ & $\begin{array}{l}-0.0170 \\
-0.0879 \\
-0.0944\end{array}$ & $\begin{array}{l}-0.0161 \\
-0.1037 \\
-0.1123\end{array}$ & $\begin{array}{l}-0.0159 \\
-0.1177 \\
-0.1268\end{array}$ \\
\hline
\end{tabular}

This work.

b A. W. Weiss, Phys. Rev. 122, 1826 (1961). This calculation includes

The terms of Eq. (12) can be given the following interpretations: The first term is essentially the SCF wavefunction, the second is a doubly excited configuration, and the third and fourth are singly excited functions. We define $\Gamma$ so that $z \equiv y / \Gamma$ is normalized, and using the property of $e^{(2,1)}$ pointed out above, Eq. (12) may be rewritten as

$$
\begin{gathered}
\Phi=\left(\gamma_{1}+\gamma_{2}\right) e^{(2,1)}\left\{a^{\prime}(1) x(2) x(3)-\Gamma^{2} a(1) z(2) z(3)\right. \\
+\delta \Gamma\left[a^{\prime}(1) x(2) z(3)-a^{\prime}(1) z(2) x(3)\right] \\
-(\langle a \mid x\rangle /\langle x \mid x\rangle) \delta \Gamma x(1) z(2) x(3)\} \\
=\Phi_{1}-\Gamma^{2} \Phi_{2}+\delta \Gamma\left(\Phi_{3}-\Phi_{4}\right)-(\langle a \mid x\rangle /\langle x \mid x\rangle) \delta \Gamma \Phi_{s}
\end{gathered}
$$

where

and

$$
\delta=\left(\gamma_{1}-\gamma_{2}\right) /\left(\gamma_{1}+\gamma_{2}\right)
$$

$$
a^{\prime}=a-(\langle a \mid x\rangle /\langle x \mid x\rangle) x \text {. }
$$

It is seen that

$$
\left\langle\Phi_{1} \mid \Phi_{3}\right\rangle=\left\langle\Phi_{1} \mid \Phi_{4}\right\rangle=0
$$

and hence these functions satisfy the orthogonality requirements of Brillouin's theorem with respect to the SCF function $\Phi_{1}$. If we set $\gamma_{2}=0, \delta=1$, and in order to have a sizable contribution of $\Phi_{2}$ in $\Phi$, the contributions of $\Phi_{3}$ and $\Phi_{4}$ must be even larger. The term $\Phi_{2}$ is the one which decreases the electronic repulsion in the correlation within the $1 s$ shell. If, however, $\Phi_{1}$ is the correct SCF function, terms $\Phi_{3}$ and $\Phi_{4}$ satisfy

$$
\left\langle\Phi_{1}|H| \Phi_{3}\right\rangle=\left\langle\Phi_{1}|H| \Phi_{4}\right\rangle=0,
$$

and the presence of these functions can only raise the calculated energy. The only way $\Phi_{3}$ and $\Phi_{4}$ could help would be to have Eq. (14) not apply, which means that $\Phi_{1}$ cannot be the SCF function. However, this possibility is also very unsatisfactory since the SCF energy is very good. Thus the restriction $\gamma_{2}=0$ requires $\Gamma$ to be very small and hence $\Gamma^{2}$ even smaller, and such a function produces essentially no correlation. If $\gamma_{1}$ and $\gamma_{2}$ are allowed to have their optimum values $\delta$ is very small $\sim 0.01$ and this fact eliminates any major contribution from the undesirable terms $\Phi_{3}$ and $\Phi_{4}$ while allowing $\Gamma$ to be larger and the desirable $\Phi_{2}$ to have a larger contribution.

The simplest type of DODS calculation of $\mathrm{Li}$ is that given by Nesbet and Watson, ${ }^{11}$ usually called spin-

${ }^{11}$ R. K. Nesbet and R. E. Watson, Ann. Phys. 9, 260 (1960). angular correlation.

E. Clementi, J. Chem. Phys. 38, 2248 (1963).

polarized Hartree-Fock, using a single Slater determinant. Löwdin ${ }^{3}$ has suggested that the reason the spinpolarized calculations provide very little correlation is the fact that a DODS, single determinant, $n$-particle function is not a pure spin state. However, a very similar analysis to that of Eq. (13) can be made for a spin-polarized determinant, and it is seen that it has the same defects with respect to Brillouin's theorem as Eq. (13) does for $\delta=1$. Goddard's function is exactly equivalent to the result of applying the spin projection operator $O_{\frac{1}{2 \frac{1}{2}}}$ to Nesbet's function. Therefore, the comparison of the correlation energy for $\mathrm{Li}$ of Nesbet and Watson, 0.00002 a.u., with that of Goddard, 0.00009 a.u., shows that removeal of the quartet state cannot alone guarantee a sizable increase in the correlation energy. It is necessary for the function to possess the flexibility to have the single excitation terms of the type of $\Phi_{3}$ and $\Phi_{4}$ multiplied by small coefficients.

It is interesting to make a conjecture concerning the result of removing the single excitation terms from the spin-polarized determinant without projecting a single spin state. It may be shown that this can be accomplished by replacing the single Slater determinant by the sum of two. Thus, in general, if the spatial orbitals $u_{1}, u_{2}, \cdots, u_{k}$ are associated with $\alpha$ spin functions and the spatial orbitals $v_{1}, v_{2}, \cdots, v_{l},(l \leq k)$ are associated with $\beta$ spin functions we may write

$\Psi=\left|u_{1} \alpha \cdots u_{k} \alpha v_{1} \beta \cdots v_{2} \beta\right|$

$$
+\left|u_{1} \beta \cdots u_{l} \beta u_{l+1} \alpha \cdots u_{k} \alpha v_{1} \alpha \cdots v_{l} \alpha\right| .
$$

The first term of $\Psi$ is just the spin-polarized determinant while the second term has the $\alpha$ 's and $\beta$ 's interchanged for the paired orbitals. This function has no single excitation. So far as the authors are aware, this type of function has not been investigated, in general, however, the special case for doublet $\mathrm{Li}$ is equivalent to the function of Pauncz et al. and to that of Eq. (13) with $\gamma_{1}=\gamma_{2}$. In this one case, removing the single excitation also removes the quartet component so that here one cannot distinguish between the two effects. In general, the function of Eq. (15) contains several different multiplicity components. Comparison of the results of Nesbet and Watson to Goddard and the present article suggests, however, that removal of the single excitations is at least as important as the removal of the extraneous spin components. 
The primitive idempotent $e^{(2,2)}$ has a factor $[I-$ (12) $][I-(34)]$ in it similar to the one in $e^{(2,1)}$. In addition, however, it has a factor $[I+(14)(23)]$ which automatically removes the single excitations in an expansion such as that of Eq. (13). Therefore, we get from Eq. (8)

$$
\begin{aligned}
\Phi \equiv & e^{(2,2)} \psi \\
= & \left(\gamma_{1}+\gamma_{2}\right) e^{(2,2)}\{u(1) x(2) x(3) u(4) \\
& -\Gamma_{1}^{2} v(1) x(2) x(3) v(4)-\Gamma_{2}^{2} u(1) y(2) y(3) u(4) \\
& +\delta \Gamma_{1} \Gamma_{2}[v(1) y(2) x(3) u(4)+u(1) x(2) y(3) v(4) \\
& -u(1) y(2) x(3) v(4)-v(1) x(2) y(3) u(4)]+\cdots\} \\
= & \Phi_{1}-\Gamma_{1}^{2} \Phi_{2}-\Gamma_{2}^{2} \Phi_{3}+\delta \Gamma_{1} \Gamma_{2} \Phi_{4}+\cdots,
\end{aligned}
$$

where

as before, and

$$
\delta=\left(\gamma_{1}-\gamma_{2}\right) /\left(\gamma_{1}+\gamma_{2}\right)
$$

$$
\begin{aligned}
& u=\frac{1}{2}(a+d), \\
& x=\frac{1}{2}(b+c), \\
& v=(a-d) / 2 \Gamma_{1},\langle v \mid v\rangle=1, \\
& y=(b-c) / 2 \Gamma_{2},\langle y \mid y\rangle=1,
\end{aligned}
$$

and we have omitted terms which can be interpreted as triple and quadruple excitations. Again $\Phi_{1}$ is close to the restricted SCF function, and $\Phi_{2}$ and $\Phi_{3}$ are the terms giving the intrashell correlations. The four individual terms in $\Phi_{4}$ are each of a type giving intershell correlation. From Table IV it is seen that $\gamma_{1} \cong \gamma_{2}$ so that $\delta$ is small and intershell correlation in these four-electron atoms is much smaller than intrashell correlation. This is physically reasonable since the $1 s$-like electrons move much more rapidly than the $2 s$-like electrons, resulting in a tendency to average out interactions in their motions. If the restriction $\gamma_{2}=0$ is applied, Eq. (16) gives a situation wherein the intershell and intrashell correlations must be more or less of the same importance. Goddard has given this case, and although his method gives the correlation energy of $\mathrm{Be}$ as 0.0142 a.u., which is considerably greater than for $\mathrm{Li}$, this is less than the value for $\mathrm{Be}^{++}, 0.0145$ a.u. Table IV shows that when $\gamma_{1}$ and $\gamma_{2}$ have their optimum values, the correlation energy in $\mathrm{Be}$ is greater than that in $\mathrm{Be}^{++}$.

The presence of a factor in $e^{(\mu)}$ which will annihilate the single excitation terms depends on the shape of tableaux, and such a factor occurs only for $\left[2^{n / 2}\right]$ tableaux, that is, in the singlet case. If, however, $\theta^{\prime} P N P$ were used for $e^{(\mu)}$, the single excitation terms would be annihilated for all cases (except $S=n / 2$ where there is no pairing at all). In spite of this, the relative simplicity of $\theta N P N$ makes us prefer to use this operator. Thus, the sum over the standard tableaux in Eq. (4) gives the wavefunction the flexibility to (a) contain very little single excitation type component and (b) at the same time allows interand intrashell correlations to take on differing importances.

The AMO procedure for alternant systems suggested by Löwdin ${ }^{3}$ (see also Ref. 12) is an approximate form of the projected Hartree-Fock procedure. In II it was shown that the projection of a single DODS determinant for the principle case $\left(M_{S}=S\right)$ is equivalent to the present treatment for

$$
\gamma_{2}=\gamma_{3}=\cdots=\gamma_{f}=0 \text {. }
$$

The $\pi$ system of the benzene molecule has been treated quite successfully with the AMO technique, but in benzene the electrons in the various shells have a much smaller spread in velocities than in $\mathrm{Be}$, therefore, the single tableaux with more or less equal inter- and intrashell correlations is much more appropriate. The standard AMO prescription calls for the projection not of the principle case but of the DODS determinant for $M_{S}=0$. Except for singlet cases this yields a result different from our $e^{(\mu)}$. In fact, such a procedure annihilates all single excitations for all odd multiplicity systems, and hence is expected to be considerably more successful than the projection of the principle determinant. ${ }^{12}$

We have collected together in Table $V$ the values of the correlation energies obtained in this study along with values from a more general treatment and the "exact" values. In the three-electron systems it is seen that radial correlation accounts for approximately one third of the total, and in the four-electron systems approximately one fifth of the total. Work is underway to include angular correlation into this treatment by the use of spatial projections.

\section{ACKNOWLEDGMENTS}

The authors would like to thank the University of Nebraska Computing Center and Professor Kenneth Smith of the Department of Computing Sciences for their aid and the contribution of time on the IBM $360 / 65$ computer.

${ }^{12}$ R. Pauncz, Alternant Molecular Orbital Method (W. B. Saunders Co., Philadelphia, Pa., 1967). 